\title{
Performance of active feedforward control systems in non-ideal, synthesized diffuse sound fields
}

\author{
Malte Misol, ${ }^{\text {a) }}$ Christian Bloch, Hans Peter Monner, and Michael Sinapius \\ Institute of Composite Structures and Adaptive Systems, German Aerospace Center, Braunschweig, Germany
}

(Received 10 September 2013; revised 30 January 2014; accepted 31 January 2014)

\begin{abstract}
The acoustic performance of passive or active panel structures is usually tested in sound transmission loss facilities. A reverberant sending room, equipped with one or a number of independent sound sources, is used to generate a diffuse sound field excitation which acts as a disturbance source on the structure under investigation. The spatial correlation and coherence of such a synthesized non-ideal diffuse-sound-field excitation, however, might deviate significantly from the ideal case. This has consequences for the operation of an active feedforward control system which heavily relies on the acquisition of coherent disturbance source information. This work, therefore, evaluates the spatial correlation and coherence of ideal and non-ideal diffuse sound fields and considers the implications on the performance of a feedforward control system. The system under consideration is an aircraft-typical double panel system, equipped with an active sidewall panel (lining), which is realized in a transmission loss facility. Experimental results for different numbers of sound sources in the reverberation room are compared to simulation results of a comparable generic double panel system excited by an ideal diffuse sound field. It is shown that the number of statistically independent noise sources acting on the primary structure of the double panel system depends not only on the type of diffuse sound field but also on the sample lengths of the processed signals. The experimental results show that the number of reference sensors required for a defined control performance exhibits an inverse relationship to control filter length. (C) 2014 Acoustical Society of America. [http://dx.doi.org/10.1121/1.4865578]
\end{abstract}

PACS number(s): 43.50.Ki, 43.40.Vn, 43.40.Qi, 43.60.Cg [SAF]

Pages: $1887-1897$

\section{INTRODUCTION}

The limited low-frequency sound transmission loss of lightweight structures, such as aircraft sidewall panels, is an important issue regarding passenger comfort. Active structural acoustic control provides a lightweight-compliant solution to the problem of low-frequency sound transmission through single or double panel structures. The experimental performance evaluation of such systems is usually done in a sound transmission loss facility by using a diffuse-soundfield excitation. This procedure must be seen as a trade-off between closeness to reality on the one hand and practicability and cost-effectiveness on the other hand. In real aircraft operation, of course, different external disturbance sources are present and the noise floor in the cabin depends on many parameters (e.g., flight attitude, propulsion system, or relative position in the cabin). A discussion of the different noise sources can be found in Schiller ${ }^{1}$ or in Wilby and Gloyna. ${ }^{2,3}$ In general it is, however, infeasible to evaluate the acoustic performance for all these different disturbance sources and circumstances. This motivates the application of a standard scenario which is described in the ISO 140-3 and the DIN EN ISO 140-3 standards. Just as in building acoustics, the structural excitation is realized by means of a diffuse sound field which is generated in a reverberation room. Although it is known that the statistical properties of the synthesized diffuse sound fields will deviate from the ideal case, potential implications on the performance of feedforward-controlled

\footnotetext{
a) Author to whom correspondence should be addressed. Electronic-mail: malte.misol@dlr.de
}

active systems have never been discussed. One reason for this might be the fact that the active control of stochastic disturbance excitations is considered as an exclusive domain of feedback algorithms. However, if coherent and sufficiently time-advanced reference signals are available, the application of feedforward control is not limited to deterministic disturbances. This was proven by the authors using the example of an aircraft-typical double panel system, where the reference sensors are placed on the primary fuselage structure and the actuators and error sensors are located on the secondary sidewall panel structure. ${ }^{4,5}$ The same experimental system is applied here in order to evaluate the implications of non-ideal statistical properties of diffuse sound fields on the performance of active feedforward-controlled structures. The research work was triggered by the observation that the number of independent components existing in the pressure field of the reverberation room or likewise in the vibration response of a structure depends on the analysis window size or the frequency resolution, respectively. The implications on the control performance are due to the fact that the frequency resolution of a feedforward controller depends on the number of filter weights, which is a free parameter in control design. To the best of the authors' knowledge, no research work has been published addressing this issue. However, as shown in the course of this paper, the implications of non-ideal conditions in reverberation rooms on the performance evaluation of active feedforwardcontrolled structures are significant.

Regarding the topic of diffuse sound fields and their generation in reverberation rooms, much theoretical and experimental research work has been published. According to 
Jacobsen and Roisin, ${ }^{6}$ the ideal diffuse sound field is defined as "[...] a sound field in an unbounded medium generated by distant, uncorrelated sources of random noise evenly distributed over all directions." Following the definition of Elliott et al., ${ }^{7}$ a diffuse sound field is induced by a superposition of an infinite number of uncorrelated plane waves. Due to the absence of interferences, the ideal diffuse sound field is homogeneous and isotropic. Regarding the practical realization of diffuse sound fields in reverberation rooms, the spatial correlation or the Schröder frequency ${ }^{8}$ are frequently used as indicators or conditions for the quality of the synthesized pressure field. Yet, the spatial coherence, which describes the number of independent components, is rarely considered in this context and has never been linked with the realization of active systems or structures. As already mentioned, most of the published research work focuses on the design and implementation of active single or double panel systems which are controlled by feedback algorithms. Past and recent work in this field has been published for example by Gardonio and Elliott, ${ }^{9}$ Engels et al., ${ }^{10}$ or Gardonio and Alujevic. ${ }^{11}$ Similar questions with special emphasis on the evaluation of different actuation principles are addressed in Bao and Pan ${ }^{12,13}$ or in Gardonio and Elliott. ${ }^{14}$ In the authors' opinion, not much research work has been published concerning the practical implementation of active structures in sound transmission loss facilities, and even fewer publications are concerned with the active feedforward control of stochastic structural vibration and the experimental evaluation of smart structures under realistic conditions.

The main body of the paper is divided into three sections. Section II starts with a theoretical discussion of the spatial correlation and coherence properties of an ideal diffuse sound field. Subsequently, the methods used for the analysis of the statistical properties of non-ideal diffuse sound fields are presented. Furthermore, a simulation model of a (pure-tone) diffuse sound field is described and its spatial correlation and coherence properties are compared to the ideal case. The section ends with a description of the reverberation room used for the synthesis of the non-ideal diffuse sound fields (generated either with one or with a multitude of independent sound sources). Here as well, the spatial correlation and coherence properties of the synthesized diffuse sound fields are analyzed and compared to the ideal case.

Section III focuses on the active feedforward control of broadband and spatially weakly correlated disturbances. First, the influence of coherence on the feedforward control performance is discussed. Subsequently, a connection between the statistical parameters of the diffuse sound field excitation and the coherence between the reference and the disturbance signals of the active feedforward control system is established. The virtual noise source theory establishes the link between the two topics of this paper: the statistical properties of the diffuse sound field and the performance of the feedforward control system. The section ends with a description of the equations used for the calculation of the finite impulse response (FIR) filter weights of the optimal causal feedforward controller.

Section IV documents the numerical and experimental investigation of the double panel system. It starts with a brief description of the experimental setup and the simulation model. Furthermore, the plot-types used for the presentation of the analysis results are described. Finally, the numerical and experimental data is evaluated regarding the relative strength of the virtual noise sources and the relative control performance of different feedforward control system configurations.

\section{CHARACTERISTICS OF IDEAL AND NON-IDEAL DIFFUSE SOUND FIELDS}

\section{A. Theory}

According to Cook et al., ${ }^{15}$ a diffuse sound field is characterized by the normalized correlation coefficient $R$,

$$
R=\frac{E[x y]}{\sqrt{E\left[x^{2}\right] E\left[y^{2}\right]}} .
$$

Equation (1) takes the expected value $E$ of two sound pressure signals $x$ and $y$, measured in the diffuse sound field at a distance $r$. The normalized correlation coefficient obtained from Eq. (1) can be compared to the theoretical value of an ideal diffuse sound field of wavenumber $k$ given by Eq. (2) (Ref. 15):

$$
R=\frac{\sin (k r)}{k r} \text {. }
$$

If the ideal diffuse sound field contains a band of frequencies ranging from $k_{1}$ to $k_{2}$, the spatial correlation is given by Eq. (3) with $k=\left(k_{1}+k_{2}\right) / 2$ (Ref. 15):

$$
R=\frac{1}{\left(k_{2} r-k_{1} r\right)} \int_{k_{1} r}^{k_{2} r} \frac{\sin (u)}{u} d u .
$$

The spatial coherence of a diffuse sound field is evaluated using the mean squared coherence $\gamma^{2}$ of the two sound pressure signals $x$ and $y$,

$$
\gamma^{2}(\omega)=\frac{\left|S_{x y}(\omega)\right|^{2}}{S_{x x}(\omega) S_{y y}(\omega)} .
$$

The cross-power spectral density is denoted by $S_{x y}$ and the power spectral densities by $S_{x x}$ and $S_{y y}$, respectively. According to Elliott et al., ${ }^{7}$ the cross-power spectral density of the pressures at two points in an ideal diffuse sound field is given by Eq. (5),

$$
S_{x y}(\omega, r)=S_{p p}(\omega) \frac{\sin (k r)}{k r} .
$$

Since the diffuse sound field is assumed to be ideal, the power spectral density $S_{p p}$ of the sound pressure is independent of position. This leads to an expression for the spatial coherence of an ideal diffuse sound field given in Eq. (6),

$$
\gamma^{2}(\omega, r)=\frac{\left|S_{x y}(\omega, r)\right|^{2}}{S_{p p}^{2}(\omega)}=\left(\frac{\sin (k r)}{k r}\right)^{2} .
$$

Hence, the spatial coherence of an ideal diffuse sound field is equal to the square of its spatial correlation. The 
validity of Eq. (6) is discussed in Jacobson and Roisin. ${ }^{6}$ In Elliott et al. ${ }^{7}$ it is mentioned that the sound field in a reverberation room can be considered as being diffuse, provided that the excitation frequencies are higher than the room's Schröder frequency. However, the spatial coherence of an ideal diffuse sound field will only be approximated well if the number of uncorrelated sound sources in the reverberation room is high enough. This condition is normally violated in reverberation rooms because of hardware limitations. Since the spatial coherence of the disturbance excitation is crucial for the design and the performance of an active structural acoustic control system with feedforward control law, it will be analyzed subsequently by means of a pure-tone diffuse sound field simulation model and by means of measurement data captured in a reverberation room.

\section{B. Evaluation of spatial correlation}

The spatial correlation of the sound pressures is evaluated for different frequency bands, each having a bandwidth of $\pm 10 \%$ of the center frequency. The results are compared to the theoretical value of the ideal diffuse sound field given in Eq. (3). The analysis is focused on low frequencies, since the bandwidth of active structures with digital controllers usually does not exceed $1000 \mathrm{~Hz}$. Furthermore, the differences between real and ideal diffuse sound fields are most prominent at lower frequencies.

For the correlation analysis, the simulated and, accordingly, the measured sound pressures are evaluated on a linear microphone array of $1.96 \mathrm{~m}$ length with quadratically increasing distances between 28 microphones. This setup was adopted from the experimental realization in order to improve the comparability between simulation and measurement. The 28 broadband sound pressure signals are bandlimited to the respective frequency band by means of a Butterworth bandpass-filter. Finally, the normalized correlation coefficient $R$ from Eq. (1) is evaluated for each of the $\left(28^{2}+28\right) / 2=406$ microphone pairings with different spacing $r$ and plotted over the normalized distance $k r$. The wavenumber $k$ corresponds to the center frequency of the band under consideration.

\section{Evaluation of spatial coherence}

The mean squared coherence of the simulated and, accordingly, the measured sound pressures is estimated for different sample lengths and compared to the theoretical value of the ideal diffuse sound field given in Eq. (6). The sound pressures used for the coherence analysis are evaluated at 29 nodes of a hexagonal array of equilateral triangles, each having a side length of $0.07 \mathrm{~m}$. This results in a total of 68 different but equally spaced microphone pairings which can be used for averaging of the mean squared coherence estimates. Again, this setup was adopted from the experimental realization in order to improve the comparability between simulation and measurement. The mean squared coherence is evaluated according to Eq. (4), using Welch's averaged and modified periodogram method. ${ }^{16}$ A Hamming window of proper size and $50 \%$ overlap was chosen for the calculation of the cross-power spectral density $S_{x y}$ and the power spectral densities $S_{x x}$ and $S_{y y}$. The resulting coherence is plotted over the normalized distance $k r$ with $r=0.07 \mathrm{~m}$.

\section{Simulation model}

Following the definition of Elliott et al., ${ }^{7}$ a diffuse sound field can be modeled by superposing a multitude of plane acoustic waves with random phase angles incident from all directions. The synthesis of the required plane waves is accomplished by means of acoustic point sources with stochastic phase angles that are evenly distributed on a halfsphere of sufficiently large dimension. In this study, a total of 300 acoustic monopoles are evenly distributed on a halfsphere with a radius of $100 \mathrm{~m}$. The mathematical expression for the sound pressure of an acoustic monopole is taken from Fahy and Gardonio $^{17}$ (p. 139). The superposed complex pressure amplitudes induced by the acoustic monopoles are evaluated on the measurement grid in order to obtain the complex diffuse sound field pressure amplitudes. However, according to Jacobsen and Roisin ${ }^{6}$ the described method equals a pure-tone model resulting in interferences of the plane waves. Since the pure-tone model deviates from the ideal, homogeneous and isotropic diffuse sound field model, its characteristics are calculated and compared to the ideal case.

Figure 1 shows the spatial correlation (a) and the spatial coherence (b) of the simulated diffuse sound field as a function of the normalized separation $k r$. Apparently, the applied pure-tone diffuse sound field model approximates the spatial correlation and coherence properties of an ideal diffuse sound field to a very high accuracy. The slight deviations between the theoretical and the simulated mean squared coherence for a sample length of $10 \mathrm{~s}$ are attributed to the lesser (a)

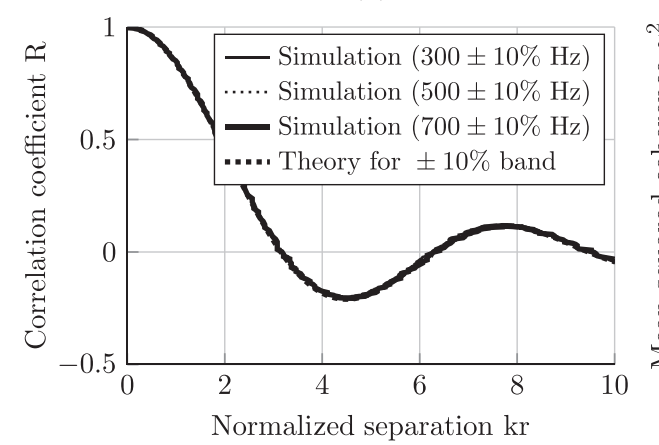

J. Acoust. Soc. Am., Vol. 135, No. 4, April 2014 (b)

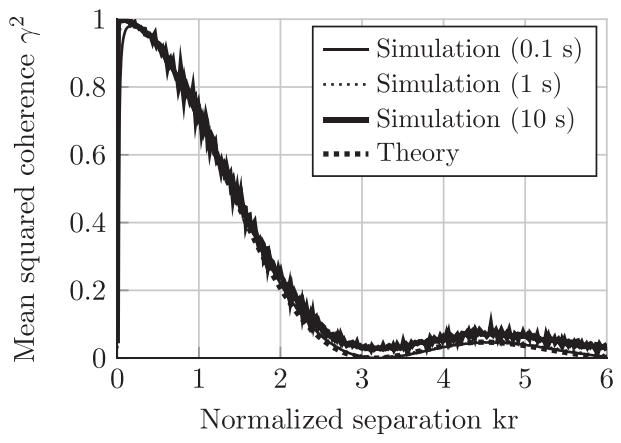

FIG. 1. (a) Spatial correlation and (b) spatial coherence of the simulated diffuse sound field (the four curves overlap). 
amount of averages available for the estimation of the periodograms. Hence, the pure-tone diffuse sound field simulation model can be applied as an ideal excitation model for the simulation-based design of active feedforward-controlled structures.

\section{E. Reverberation room}

Real diffuse sound fields are usually generated in reverberation rooms excited by at least one or a few statistically independent sound sources. In this study, a reverberation room of $\approx 200 \mathrm{~m}^{3}$ with a mean reverberation time of $\approx 5 \mathrm{~s}$ (averaged over third-octave bands from 80 to $5000 \mathrm{~Hz}$ ) was used. The room has a Schröder frequency of $\approx 125 \mathrm{~Hz}$ and fulfills the ISO 3741 standard for frequencies above $100 \mathrm{~Hz}$. The excitation of the reverberation room was realized by means of either an omnidirectional dodecahedron sound source with 12 shunted electrodynamic loudspeakers (all excited by the same signal) or of 10 independent electrodynamic loudspeakers, which are part of a loudspeaker array. The dodecahedron sound source was driven by a bandlimited white noise signal with a frequency range of $0-5000 \mathrm{~Hz}$, the loudspeaker array by 10 uncorrelated, bandlimited white noises ranging from 80 to $5000 \mathrm{~Hz}$. The sound pressure was measured using $1 / 4$ in. $\mathrm{ICP}^{\circledR}$ microphones of the type PCB $130 \mathrm{D} 21$.

\section{Single sound source}

Figure 2(a) shows the spatial correlation of the diffuse sound field excited by a single sound source as a function of the normalized separation $k r$. Especially for the lower frequency bands, the correlation is higher as would be expected from the ideal case. The deviations are attributed to the fact that there is only one noise source present in the reverberation room. As can be seen in Fig. 2(b), the coherence of the diffuse sound field rises with increasing sample length and approaches one if the analysis window exceeds the reverberation time. The resulting interferences and standing waves deteriorate the isotropy and homogeneity of the diffuse sound field, which is reflected in larger deviations of the spatial correlation compared to the ideal case. However, the deviations between real and theoretical spatial correlation are surprisingly high given that all frequencies lie above the Schröder frequency and, even for the lowest frequency at $270 \mathrm{~Hz}$, the number of room modes within the half-power bandwidth is $\approx 13$. The half-power bandwidth was approximated by
$2 \sigma=-2 \ln (0.001) / T_{60} \approx 2.76 \mathrm{~Hz}$, with the average reverberation time $T_{60}=5 \mathrm{~s}$. The modal density at frequency $f$ in a reverberation room of volume $V$ is given by ${ }^{18}$

$$
\frac{\Delta M}{\Delta f} \approx \frac{d M}{d f}=\frac{4 \pi}{c}\left(\frac{f}{c}\right)^{2} V .
$$

Figure 2(b) shows the corresponding spatial coherence as a function of the normalized separation $k r$. As already noted, the deviation between real and theoretical coherence rises with increasing sample length. This behavior is explained by the fact that the reverberation room acts as an acoustic energy storage. If the excitation is stochastic, a short sequence of sound pressure signals (sample length $\ll T_{60}$ ) contains a large amount of uncorrelated sound energy. Since the amount of stored energy is proportional to the reverberation time, the influence of uncorrelated components decreases for larger sample lengths. As a consequence, the spatial coherence rises with increasing sample length as shown in Fig. 2. Jacobsen and Roisin ${ }^{6}$ provide an alternative explanation based on the bandwidth of the excitation signal and the characteristic dimension of the reverberation room.

\section{Multiple sound sources}

Figure 3(a) shows the spatial correlation of the diffuse sound field excited by 10 uncorrelated sound sources as a function of the normalized separation $k r$. Apart from the lowest frequency band, the spatial correlation of the real and the ideal diffuse sound field is almost identical. The higher agreement is explained by the use of multiple uncorrelated sound sources which couple differently and statistically independently to the room modes. This results in a more isotropic and homogenous sound field which better approximates the characteristics of the ideal diffuse sound field. The existence of multiple uncorrelated sound sources is also reflected in the spatial coherence shown in Fig. 3(b). However, the coherence rises for increasing sample lengths which indicates that the theoretical number of independent sources of an ideal diffuse sound field has still not been reached.

\section{ACTIVE FEEDFORWARD CONTROL}

Figure 4 shows the general scheme of a feedforward control system. The control signals $\mathbf{u}$ are generated by filtering the reference signals $\mathbf{x}$ through the feedforward (a)

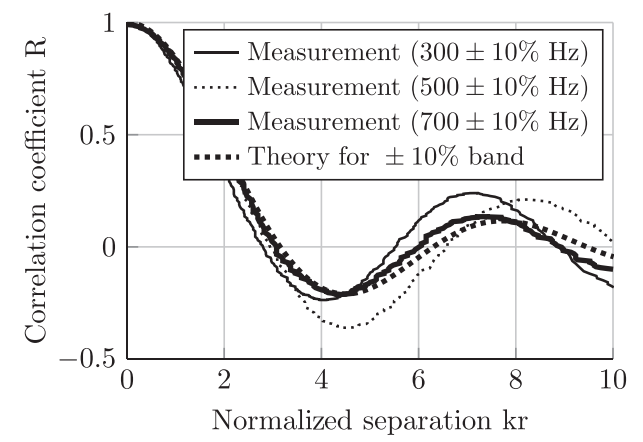

(b)

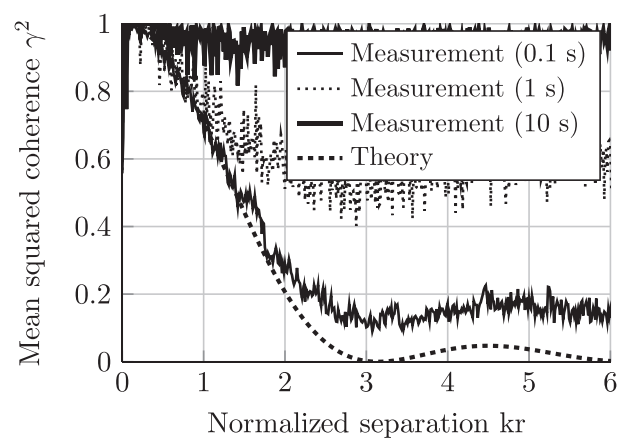

FIG. 2. (a) Spatial correlation and (b) spatial coherence of the diffuse sound field in the reverberation room generated with a single sound source. 
(a)

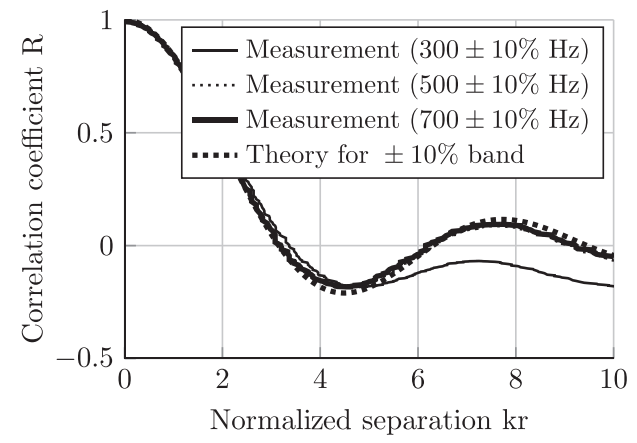

(b)

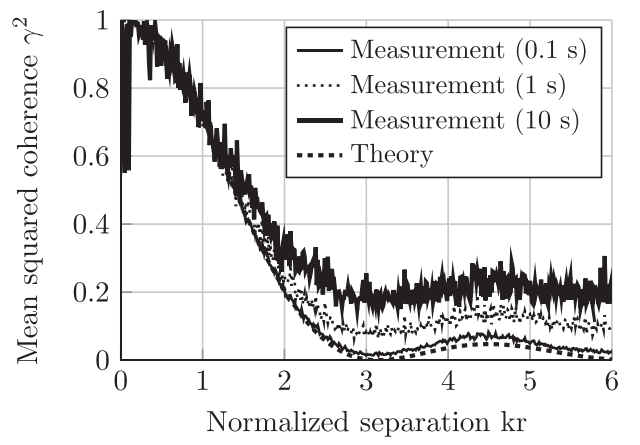

FIG. 3. (a) Spatial correlation and (b) spatial coherence of the diffuse sound field in the reverberation room generated with 10 uncorrelated sound sources. controller $\mathbf{W}$. The secondary path $\mathbf{G}$ describes the dynamics between the controller output and the error sensors. The error signal $\mathbf{e}$ is the sum of the filtered control signals $\mathbf{y}$ and the disturbance signals $\mathbf{d}$.

\section{A. Parameters on feedforward control performance}

The performance of an active feedforward controller is largely influenced by the availability of time-advanced and coherent reference signals. The first requirement is related to the causality of the feedforward control system. For broadband excitations like a diffuse sound field or a turbulent boundary layer, the causality constraint is an important issue. Yet, this study focuses on the second requirement, as the implications of non-ideal diffuse sound fields on the coherence and the performance of a feedforward control system are crucial.

In order to assess the influence of coherence on the performance of a feedforward control system, a performance metric must be defined. Equation (8) provides a typical cost function leading to an expression of the unconstrained feedforward controller in the frequency domain. For the sake of clarity, the discrete frequency parameter $\kappa$ (as defined in Sec. III C) is omitted in the present and in the following section. The formulation is taken from Elliott, ${ }^{19}$

$$
J=\operatorname{trace} E\left[\mathbf{e} \mathbf{e}^{H}\right]=\mathbf{S}_{\mathbf{e e}} .
$$

The error signal spectra are contained in the column vector $\mathbf{e}$ and the expected value of its outer product equals the power spectral density matrix $\mathbf{S}_{\mathrm{ee}}$. According to Elliott, ${ }^{19}$ the minimum value of the cost function for a feedforward control system with an equal number of actuators and error sensors is given by

$$
J_{\min }=\operatorname{trace}\left[\mathbf{S}_{\mathbf{d d}}-\mathbf{S}_{\mathbf{x d}} \mathbf{S}_{\mathbf{x x}}^{-1} \mathbf{S}_{\mathbf{x d}}^{H}\right] .
$$

A connection between control performance and coherence is provided by Minkoff ${ }^{20}$ for the special case of a system with $K \equiv M \equiv L \equiv 2$. For reasons of clarity, no virtual transfer functions are defined between reference and disturbance signals, as had been the case in the formula provided by Minkoff. Therefore, the cost function described in Eqs. (10) and (11) slightly differs from the original one,

$$
\begin{aligned}
J_{\min }= & S_{d_{1} d_{1}}\left(1-\frac{\gamma_{d_{1} x_{1}}^{2}+\gamma_{d_{1} x_{2}}^{2}}{1-\gamma_{x_{1} x_{2}}^{2}}\right) \\
& +2 \operatorname{Re}\left[\frac{S_{x_{1} x_{2}}}{S_{x_{1} x_{1}} S_{x_{2} x_{2}}} \frac{S_{x_{1} d_{1}} S_{x_{1} d_{2}}^{*}}{1-\gamma_{x_{1} x_{2}}^{2}}\right] \cdots \\
& +S_{d_{2} d_{2}}\left(1-\frac{\gamma_{d_{2} x_{2}}^{2}+\gamma_{d_{2} x_{1}}^{2}}{1-\gamma_{x_{1} x_{2}}^{2}}\right) \\
& +2 \operatorname{Re}\left[\frac{S_{x_{1} x_{2}}}{S_{x_{1} x_{1}} S_{x_{2} x_{2}}} \frac{S_{x_{2} d_{1}} S_{x_{2} d_{2}}^{*}}{1-\gamma_{x_{1} x_{2}}^{2}}\right] \\
= & S_{d_{1} d_{1}}+S_{d_{2} d_{2}}-\frac{1}{1-\gamma_{x_{1} x_{2}}^{2}} \cdots \\
& +\left\{S_{d_{1} d_{1}}\left(\gamma_{d_{1} x_{1}}^{2}+\gamma_{d_{1} x_{2}}^{2}\right)-2 \operatorname{Re}\left[\frac{S_{x_{1} x_{2}} S_{x_{1} d_{1}} S_{x_{1} d_{2}}^{*}}{S_{x_{1} x_{1}} S_{x_{2} x_{2}}}\right] \ldots\right. \\
& \left.+S_{d_{2} d_{2}}\left(\gamma_{d_{2} x_{2}}^{2}+\gamma_{d_{2} x_{1}}^{2}\right)-2 \operatorname{Re}\left[\frac{S_{x_{1} x_{2}} S_{x_{2} d_{1}} S_{x_{2} d_{2}}^{*}}{S_{x_{1} x_{1}} S_{x_{2} x_{2}}}\right]\right\}
\end{aligned}
$$

If one assumes that the feedforward controller does not increase the value of the cost function, the third summand of Eq. (11) has to be positive. Consequently, $J_{\min }$ will reach its minimum if the mutual coherence between the reference signals $\gamma_{x_{1} x_{2}}^{2}$ (and with it $S_{x_{1} x_{2}}$ ) vanishes and the multiple coherence between the disturbance and the reference signals $\gamma_{d_{i} \mathbf{x}}^{2}$ is equal to one. The multiple coherence cannot exceed unity and for the case $\gamma_{x_{i} x_{j}}^{2} \equiv 0$ it holds ${ }^{21}$

$$
\gamma_{d_{i} \mathbf{x}}^{2}=\sum_{k=1}^{K} \gamma_{d_{i} x_{k}}^{2} \leq 1
$$

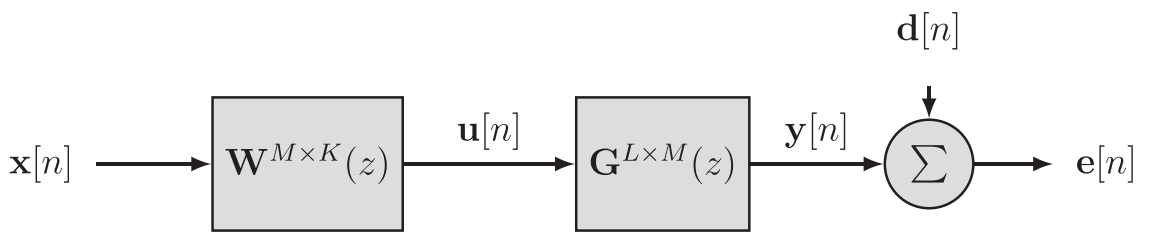

FIG. 4. Block diagram of an active feedforward control system with $K$ reference sensors, $M$ actuators and $L$ error sensors. 


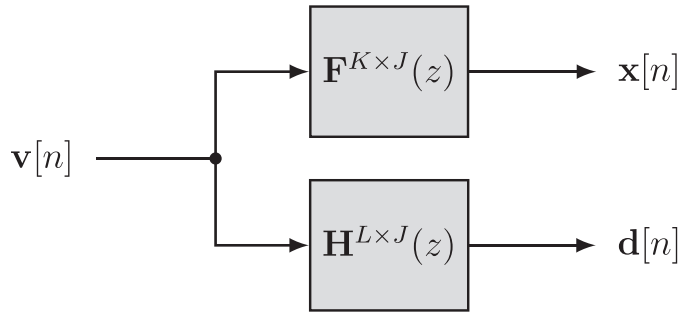

FIG. 5. Synthesis of reference signals $\mathbf{x}$ and disturbance signals $\mathbf{d}$ from virtual noise sources $\mathbf{v}$.

However, due to the spatial correlation of the sound pressure in a diffuse sound field, the reference signals will not be statistically independent and the number and positioning of the reference sensors must be optimized. This also applies to non-acoustic reference signals acquired on a vibrating structure under diffuse sound field excitation. Further insight into the connection between the statistical properties of the excitation and the feedforward control performance is gained by applying the virtual noise source theory.

\section{B. Virtual noise source theory}

The concept of virtual noise sources described in Akiho et $a l^{22}$ provides a method to identify the number of statistically independent components in a sound or vibration field. In Elliott et al., ${ }^{7}$ this method is used to calculate the density of uncorrelated components in an ideal diffuse sound field. In this study, the theory is applied to structural reference signals measured on the primary structure $P$ of a double panel system (see Fig. 6) excited by a diffuse sound field. The number of independent components in the vibration field of $P$, which act as disturbance sources of the secondary structure $S$, corresponds to the number of dominant eigenvalues of the power spectral density matrix,

$$
\mathbf{S}_{\mathbf{x x}}=E\left[\mathbf{x x}^{H}\right] .
$$

Whether or not an eigenvalue must be considered as dominant, depends on the prescribed control performance. In order to impose a tolerance limit on the eigenvalues, their physical meaning and influence on the multiple coherence of the disturbance and the reference signals has to be assessed.

Figure 5 shows the connection between the virtual noise sources $\mathbf{v}$, the reference signals $\mathbf{x}$ and the disturbance signals d. Accordingly, the power spectral density matrix of Eq. (13) can be rewritten as

$$
\mathbf{S}_{\mathbf{x x}}=E\left[\mathbf{x} \mathbf{x}^{H}\right]=E\left[\mathbf{F} \mathbf{v} \mathbf{v}^{H} \mathbf{F}^{H}\right]=\mathbf{F} \underbrace{E\left[\mathbf{v} \mathbf{v}^{H}\right]}_{\mathbf{S}_{\mathbf{v} v}=\Sigma} \mathbf{F}^{H} .
$$

Since the virtual noise sources are uncorrelated, Eq. (14) takes the form of an eigenvector/eigenvalue decomposition. The matrix $\mathbf{F}$ contains the eigenvectors and the diagonal matrix $\Sigma$ contains the eigenvalues of $\mathbf{S}_{\mathbf{x x}}$. It follows, that the eigenvalues $\sigma_{i}=\Sigma_{i i}$ of $\mathbf{S}_{\mathbf{x x}}$ are equal to the power spectral densities $E\left[v_{i}^{*} v_{i}\right]$ of the virtual noise sources. Assuming that the magnitudes of the frequency response functions $H_{d_{i} v_{j}}, j=1, \ldots, J$ are identical or at least similar, the relative contribution of a virtual noise source to the multiple coherence is identical or similar to its power spectral density. The required value of multiple coherence depends on and can be derived from the cost function of the feedforward controller. Based on the multiple coherence value, a tolerance bound on the eigenvalues and the minimum number of reference sensors can be derived. Yet, due to the influence of structural dynamics and suboptimal reference sensor positions, the required number of reference sensors may exceed the theoretical minimum value.

\section{Optimal causal feedforward control}

The calculation of the optimal causal feedforward controller is based on a matrix formulation for the error vector which permits an explicit expression for the vector of optimal filter coefficients. The benefits of this method are its inherent causality, the specification of the number of filter weights and the possibility to include control effort into the performance metric. The derivation and notation is adopted from Elliott. ${ }^{19}$ The vector of optimal finite impulse response (FIR) filter weights is given by

$$
\mathbf{w}_{\mathrm{opt}}=-\left(\mathbf{R}_{\tilde{\mathbf{x}} \tilde{\mathrm{x}}}+\beta \mathbf{I}\right)^{-1} \mathbf{R}_{\tilde{\mathbf{x}} \mathbf{d}} .
$$

The calculation of the auto-correlation matrix $\mathbf{R}_{\tilde{x} \tilde{x}}$ of the filtered reference signals $\tilde{\mathbf{x}}$ and the cross-correlation vector $\mathbf{R}_{\tilde{x} \mathbf{d}}$ is performed in the frequency domain. This improves the numerical efficiency and permits the use of frequencyresponse-data models instead of state-space models which ensures stability even for ill-conditioned system models

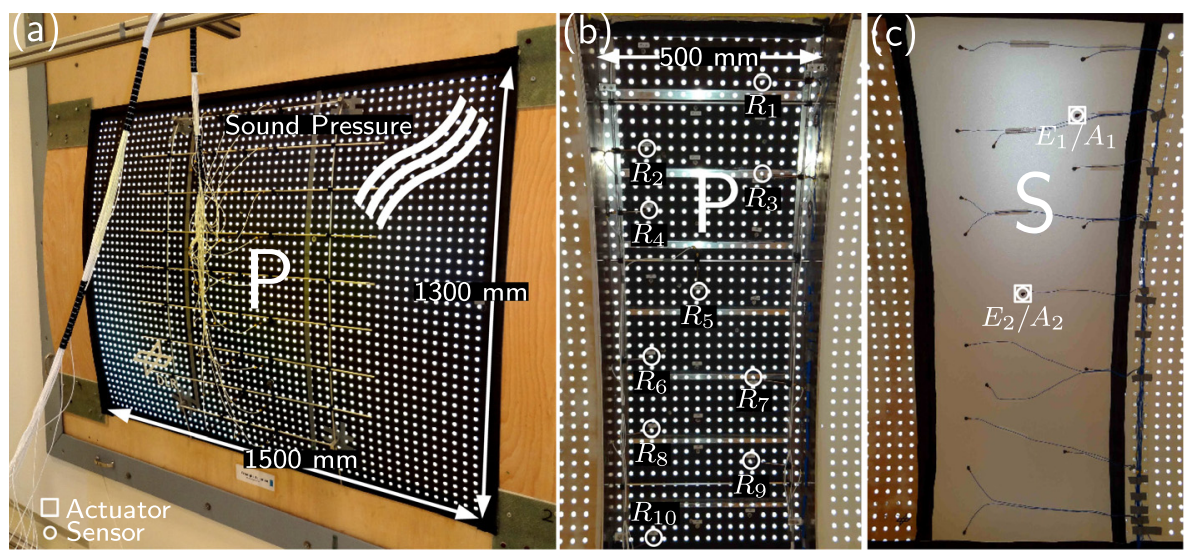

FIG. 6. (Color online) Experimental setup of the active double panel system with primary fuselage structure $\mathrm{P}$ and secondary lining structure $\mathrm{S}$ seen from the reverberation room (a) and from the semi-anechoic room (b) and (c) of the sound transmission loss facility. 
(e.g., from finite element method). Due to its block-Toeplitz structure, the auto-correlation matrix is highly redundant and iterative methods are available to solve for the optimal filter weights. ${ }^{23}$ The regularization factor $\beta \geq 0$ penalizes the control effort and improves the numerical stability and robustness of the controller.

The auto-correlation block-Toeplitz matrix of the filtered reference signals is given in Eq. (16),

$$
\mathbf{R}_{\tilde{\mathrm{x}} \tilde{\mathrm{x}}}=\left[\begin{array}{cccc}
\mathbf{R}_{\tilde{\mathrm{x}} \tilde{\mathrm{x}}}[0] & \mathbf{R}_{\tilde{\mathrm{x}} \tilde{\mathrm{x}}}[-1] & \cdots & \mathbf{R}_{\tilde{\mathrm{x}} \tilde{\mathrm{x}}}[1-I] \\
\mathbf{R}_{\tilde{\mathrm{x}} \tilde{\mathrm{x}}}[1] & \mathbf{R}_{\tilde{\mathrm{x}} \tilde{\mathrm{x}}}[0] & \cdots & \vdots \\
\vdots & \ldots & \ddots & \vdots \\
\mathbf{R}_{\tilde{\mathrm{x}} \tilde{\mathrm{x}}}[I-1] & \ldots & \cdots & \mathbf{R}_{\tilde{\mathrm{x}} \tilde{\mathrm{x}}}[0]
\end{array}\right]
$$

The individual blocks of $\mathbf{R}_{\tilde{x} \tilde{x}}$ are given by

$$
\mathbf{R}_{\tilde{x} \tilde{x}}[\tau]=\sum_{l=1}^{L}\left[\begin{array}{cccccc}
r_{\tilde{x}_{l 1} \tilde{x}_{l 11}}[\tau] & r_{\tilde{x}_{l 11} \tilde{l}_{l 12}}[\tau] & \cdots & r_{\tilde{x}_{l 11} \tilde{x}_{l 1 K}}[\tau] & \cdots & r_{\tilde{x}_{l 11} \tilde{l}_{l M K}}[\tau] \\
r_{\tilde{x}_{l 12} \tilde{x}_{l 11}}[\tau] & r_{\tilde{x}_{l 12} \tilde{x}_{l 12}}[\tau] & \cdots & \cdots & \cdots & \vdots \\
\vdots & \cdots & \ddots & \ldots & \ldots & \vdots \\
r_{\tilde{x}_{l 1 K} \tilde{x}_{l 11}}[\tau] & \ldots & \cdots & \ddots & \ldots & \vdots \\
\vdots & \cdots & \cdots & \cdots & \cdots & \vdots \\
r_{\tilde{x}_{l M K} \tilde{x}_{l 11}}[\tau] & \cdots & \cdots & \cdots & \cdots & r_{\tilde{x}_{l M K} \tilde{x}_{l M K}}[\tau]
\end{array}\right]
$$

Equation (18) gives the cross-correlation vector of the filtered reference signals $\tilde{\mathbf{x}}$ and disturbance signals $\mathbf{d}$,

$$
\mathbf{R}_{\tilde{\mathbf{x}} \mathbf{d}}=\left[\begin{array}{llll}
\mathbf{R}_{\tilde{\mathbf{x}} \mathbf{d}}[0] & \mathbf{R}_{\tilde{\mathbf{x}} \mathbf{d}}[-1] & \cdots & \mathbf{R}_{\tilde{\mathbf{x}} \mathbf{d}}[1-I]
\end{array}\right]^{T} .
$$

The sub-vectors of $\mathbf{R}_{\tilde{x} \mathbf{d}}$ are given by

$$
\mathbf{R}_{\tilde{x} \mathbf{d}}[\tau]=\sum_{l=1}^{L}\left[\begin{array}{llllll}
r_{\tilde{x}_{l 11} d_{l}}[\tau] & r_{\tilde{x}_{l 12} d_{l}}[\tau] & \cdots & r_{\tilde{x}_{l 1 K} d_{l}}[\tau] & \cdots & r_{\tilde{x}_{l M K} d_{l}}[\tau]
\end{array}\right]^{T}
$$

As noted above, the calculation of the required crosscorrelations is performed in the frequency domain. The cross-power spectra $S_{\tilde{x}_{l m k} \tilde{x}_{q r s}}$ are obtained from the filtered reference signal spectra in Eq. (21) and the cross-correlation results from an inverse discrete Fourier transform (IDFT). The calculation of $r_{\tilde{x}_{l m k} d_{l}}$ is carried out analogously,

$$
\begin{aligned}
r_{\tilde{x}_{l m k} \tilde{x}_{q r s}}(\tau) & =\operatorname{IDFT}\left\{S_{\tilde{x}_{l m k} \tilde{x}_{q r s}}\right\} \\
& =\operatorname{IDFT}\left\{E\left[\tilde{x}_{l m k}^{*}(\kappa) \tilde{x}_{q r s}(\kappa)\right]\right\} .
\end{aligned}
$$

The parameter $\kappa=0,1, \ldots, N-1$ describes the $\kappa$ th frequency bin with discrete frequency $\omega_{\kappa}=\kappa \omega T / N$. The discrete-time step-size is denoted by $T$. The connection between the number of FIR filter weights $I$ and the number of discrete frequency lines $N$ is $N=2 I$,

$$
\tilde{x}_{l m k}(\kappa)=G_{l m}(\kappa) x_{k}(\kappa) \text {. }
$$

\section{DOUBLE PANEL SYSTEM WITH ACTIVE SECONDARY STRUCTURE}

\section{A. System and methodology}

In this study, an aircraft-typical double panel system is used for the implementation of the active feedforward control system. As shown in Fig. 6, the system consists of a curved and stiffened carbon-fiber-reinforced plastics (CFRP) fuselage structure $P$ and an off-the-shelf aircraft sidewall panel $S$. The fuselage structure $P$ is excited by means of a non-ideal diffuse sound field which is synthesized according to Sec. IIE. The microphone array mounted in front of $P$ is not used for the experiments documented in this paper. As shown in Fig. 6, most structural surfaces are covered with a regular grid of laser reflection points. These points consist of a very thin film and it can be assumed, that their influence und the structural vibration and sound radiation is negligible. The fuselage structure $P$ is augmented with 10 reference accelerometers $R_{1}$ to $R_{10}$, which measure the structural vibration induced by the diffuse sound field. The optimal reference sensors are chosen from a greater subset by minimizing the mutual coherence of the reference signals and maximizing the multiple coherence of reference and disturbance signals. This procedure is justified by the analysis provided in Sec. III A of this text. The investigations are carried out by using two pairs of error sensors $E_{i}$ (accelerometers) and actuators $A_{i}$ (inertial mass exciters), which are mounted on the secondary structure $S$. The restriction to two actuator-sensor pairs is reasonable as it reduces the numerical effort without compromising the generality of the derived results. The positions of the error sensors on $\mathrm{S}$ are chosen in order to maximize the observability of the structural vibration. Each actuator is applied collocated to the 


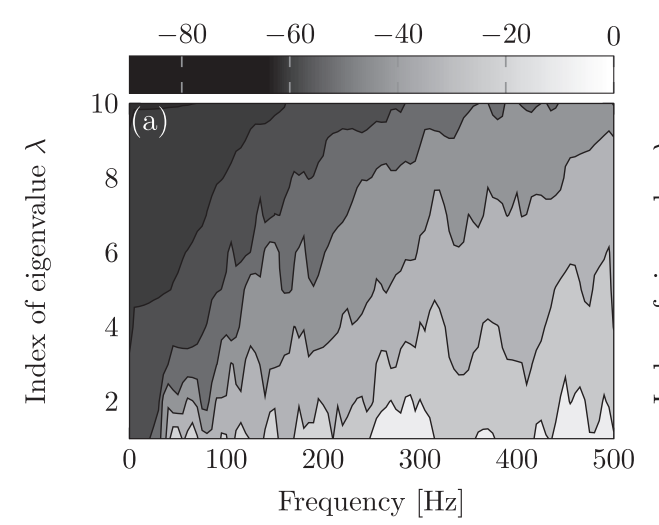

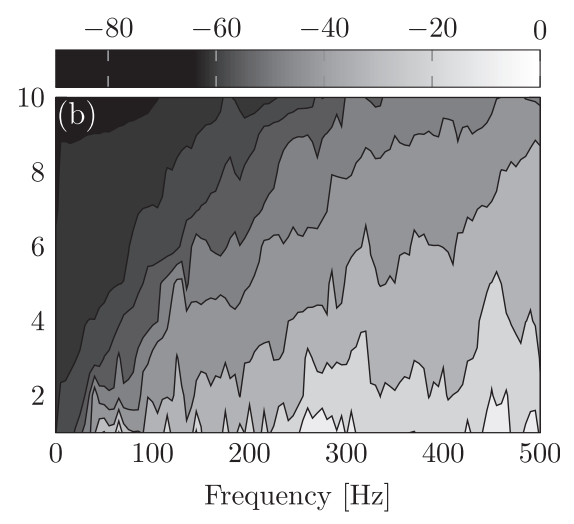

FIG. 7. Normalized eigenvalue levels of the power spectral density matrix $\mathbf{S}_{\mathbf{x x}}$ of the simulated reference signals (10 accelerations) calculated with an analysis window of $0.2 \mathrm{~s}$ (a) and $3 \mathrm{~s}$ (b). corresponding error sensor on the backside of the sidewall panel. This minimizes the delay in the secondary paths $G_{i i}$ with $i=1,2$ and thus increases the causality margin of the feedforward control system. ${ }^{4}$

The simulations are carried out for a simplified, generic double panel system consisting of two flat panels coupled by an enclosed fluid. The geometrical and material properties of the generic system are similar to the real system, yet the horizontal width of $P$ is limited to $0.5 \mathrm{~m}$ and thus equals the distance between two frames. Further details on the modeling of the generic double panel system can be found in Misol and Hesse. ${ }^{5}$

The numerical and experimental results provided in the following are intended to clarify the relationship between the number of dominant virtual noise sources and the performance of optimal causal feedforward control. The results of the analysis are provided by means of two different plottypes.

First, a contour plot of the eigenvalues of the power spectral density matrix $\mathbf{S}_{\mathbf{x x}}$ is used in order to evaluate the strength of the virtual noise sources. It was shown in Sec. III B, that the power spectral densities of the virtual noise sources are identical to the eigenvalues of $\mathbf{S}_{\mathbf{x x}}$.

According to Eq. (13), the power spectral density matrix $\mathbf{S}_{\mathbf{x x}}$ equals the expectation of the dyadic product of the vector of the reference signal spectra $\mathbf{x}$. The required reference signal spectra are obtained either from the simulation models of the diffuse sound field and the double panel system or from measurement data of the double panel system. The sampling frequency $F_{s}=1 / T$ was set to $1000 \mathrm{~Hz}$ and the frequency resolution equals $5 \mathrm{~Hz}$ for the short analysis window and $1 / 3 \mathrm{~Hz}$ for the long analysis window. The measurement data results from a synchronous sampling of the signals of the reference sensors $R_{1}$ to $R_{10}$ for both types of non-ideal diffuse-sound-field excitations described in Sec. IIE The numerical data is obtained from a doublestage process. First, the complex pressure amplitudes of the pure-tone diffuse sound field (described in Sec. IID) are calculated for all frequency bins and at all nodes of the finite-element model of the primary structure (fuselage). Second, the pressure spectra are filtered through a frequency-response-data model of the double panel system. This process is repeated several times, in order to obtain enough statistically independent data to evaluate the expectation operator in Eq. (13).
The magnitudes of the contour plots in Figs. 7, 9, and 11 correspond to the normalized eigenvalue levels $\lambda_{i}(\kappa)$ $=10 \log _{10}\left(\sigma_{i}(\kappa) / \sigma_{\max }\right) \mathrm{dB}$. The spacing of the contour lines is $10 \mathrm{~dB}$. Two different analysis window sizes are chosen, one well below and one in the range of the reverberation time. As is known from Sec. IIE, the sample length has a significant influence on the spatial coherence and thus influences the number of dominant virtual noise sources.

Second, in Figs. 8, 10, and 12, the control performance of a long FIR filter with 3000 filter taps is compared to that of a short FIR filter with 200 filter taps. It is assumed from Sec. II E, that the FIR filter length will strongly influence the performance of the feedforward control system, since it determines the duration over which the filter correlates.

The FIR filter weights are calculated according to Eq. (15). As described in Sec. III C, the required correlation matrices $\mathbf{R}_{\tilde{\mathrm{x}} \tilde{\mathrm{x}}}$ and $\mathbf{R}_{\tilde{\mathrm{x}} \mathbf{d}}$ are obtained from the reference signal spectra $x_{k}$, the frequency response functions $G_{l m}$ and the disturbance signal spectra $d_{l}$. Again, the required signal spectra and frequency response functions are obtained either from the simulation models of the diffuse sound field and the double panel system or from measurement data of the double panel system. The frequency response functions $G_{l m}$ of the secondary path incorporate the signal propagation delays induced by the low-pass filters, the zero-order hold and the digital signal processing system. As before, the sampling frequency $F_{s}=1 / T$ was set to $1000 \mathrm{~Hz}$.

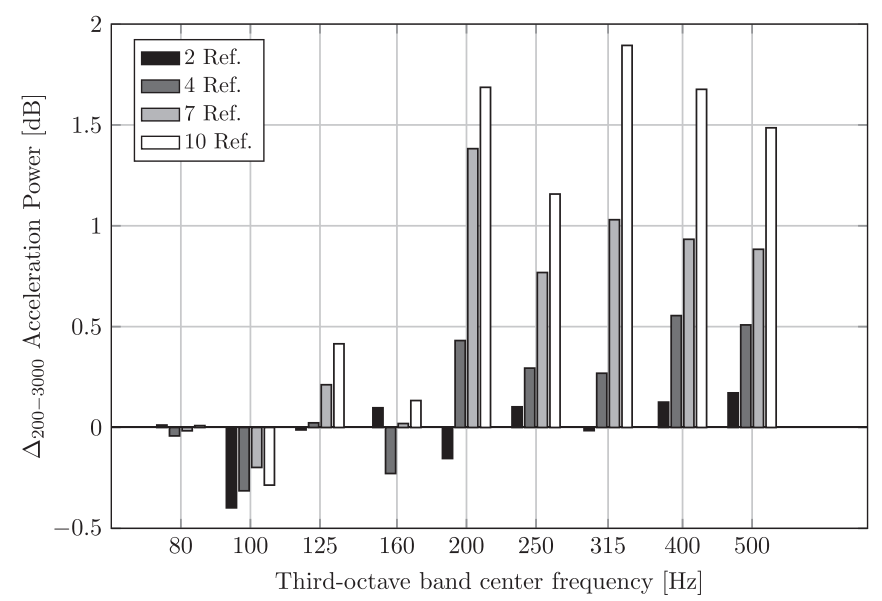

FIG. 8. Simulated difference in control performance of a feedforward controller with 3000 and with 200 FIR filter taps dependent on the number of reference sensors. 

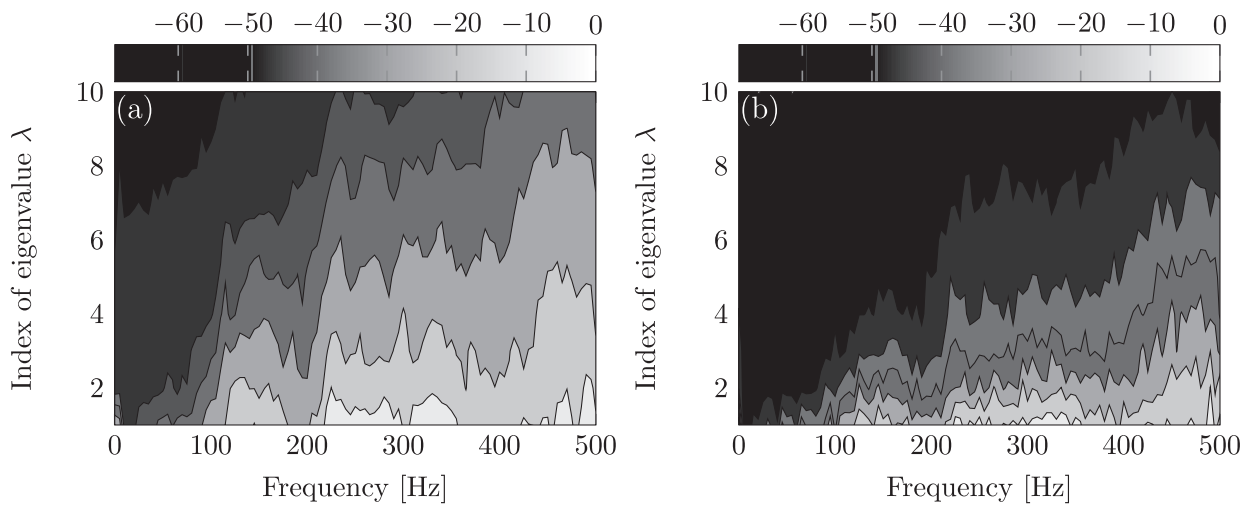

FIG. 9. Normalized eigenvalue levels of the power spectral density matrix $\mathbf{S}_{\mathbf{x x}}$ of the measured reference signals (10 accelerations) calculated with an analysis window of $0.2 \mathrm{~s} \mathrm{(a)} \mathrm{and} 3 \mathrm{~s}$ (b) with a single sound source in the reverberation room.

The relative control performance $\mathbf{S}_{\mathrm{ee}, 200}-\mathbf{S}_{\mathrm{ee}, 3000}$ $=\Delta_{200-3000}$ is evaluated in terms of the average power spectral densities of the error signals. Different reference sensor configurations are considered in order to observe the interdependencies between control performance, FIR filter length, number of reference sensors and type of diffuse sound field excitation. It must be noted, that the FIR filter sizes correspond to the sample lengths of the analysis windows used for the calculation of the power spectral density matrix $\mathbf{S}_{\mathbf{x x}}$. The configuration with 2 references uses $R_{3}$ and $R_{6}$, the configuration with four references uses $R_{1}, R_{4}, R_{6}$, and $R_{9}$ and the configuration with seven references uses $R_{1}, R_{2}, R_{3}, R_{5}$, $R_{6}, R_{7}$, and $R_{10}$. It must also be noted, that the reference sensor positions are optimized for the full set of 10 reference sensors which implies a suboptimal positioning for the other configurations. Due to this fact, the number of reference sensors required for a high multiple coherence usually exceeds the number of dominant virtual noise sources.

\section{B. Simulation}

According to Fig. 7, the dependency of the eigenvalue levels on sample length is very weak. This result is in accordance with the spatial coherence shown in Fig. 1(b), which was only slightly increased for larger analysis windows. As noted above, the slight dependence on sample length is attributed to the smaller number of averages

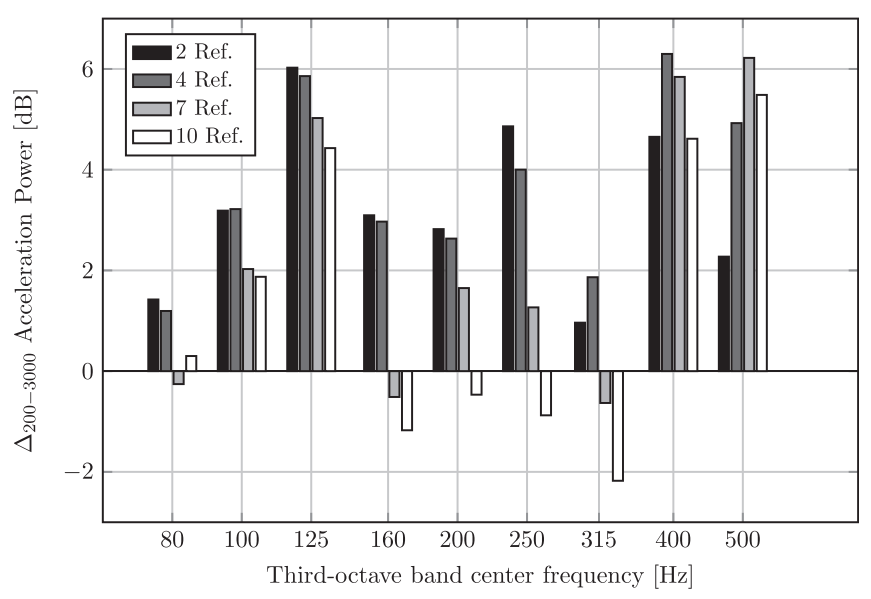

FIG. 10. Measured difference in control performance of a feedforward controller with 3000 and with 200 FIR filter taps dependent on the number of reference sensors with a single sound source in the reverberation room. available for the spectral averaging. Figure 7 further shows that the eigenvalue levels increase with frequency, which according to Elliott et $a l^{7}$ is typical for an ideal diffuse sound field. The monotonicity of the contour lines is disturbed due to the influence of structural resonances and antiresonances. The number of dominant virtual noise sources can be inferred from Fig. 7 by counting the number of eigenvalues that are located within the span of a defined number of contour lines. If two contour lines are considered, the span equals $20 \mathrm{~dB}$ which corresponds to $99 \%$ of the virtual noise source power. According to this definition, approximately eight eigenvalues are dominant at $500 \mathrm{~Hz}$. Hence, the use of eight optimally placed reference sensors should permit a multiple coherence of more than 99\%. However, due to the influence of causality and suboptimal placement of reference sensors, the achievable control performance cannot be inferred directly from the multiple coherence alone.

For reasons of brevity, the absolute control performance in terms of the error sensors power spectral densities is not provided. Instead, the maximum average third-octave-band acceleration power levels serve as reference for the relative control performances shown in Figs. 8, 10, and 12. For the system under consideration in Sec. IV B, the maximum reduction in third-octave-band acceleration power level (averaged over the error sensors $E_{1}$ and $E_{2}$ ) amounts to $\approx 20 \mathrm{~dB}$ (in the $160 \mathrm{~Hz}$ third-octave band). Hence, the relative control performance shown in Fig. 8 amounts to only $\approx 10 \%$ of the $\mathrm{dB}$-value of the maximum third-octave-band power reduction (or $\approx 1.6 \%$ on a linear scale) which is rather small compared to the experimental results with non-ideal diffuse sound fields. The increase in relative control performance at $200 \mathrm{~Hz}$ and above is probably due to the longer FIR filters, which achieve a better modeling of the system dynamics.

\section{Experiment}

As is shown in Fig. 9, the dependency of the eigenvalue levels on sample length for a non-ideal diffuse sound field with a single sound source in the reverberation room is much stronger compared to the previous case. Again, this behavior is in accordance with the spatial coherence shown in Fig. 2(b), which converges to the theoretical value of the ideal diffuse sound field for very small analysis windows and which tends to one for the opposite case. It is thus assumed that a feedforward controller with short FIR filters will 

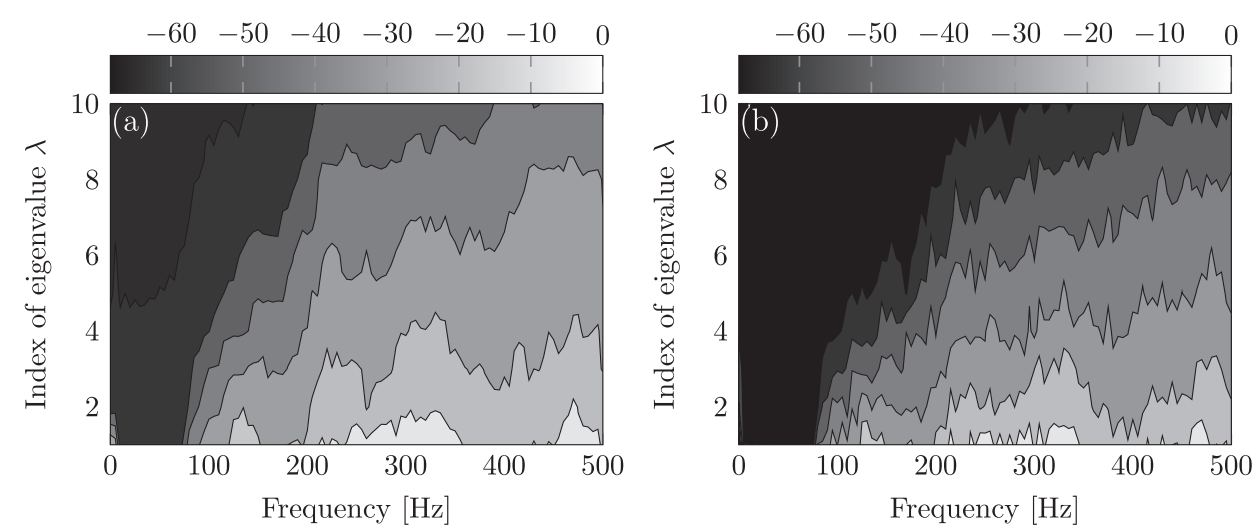

observe an almost ideal diffuse sound field and hence will require much more reference sensors as a feedforward controller with long FIR filters that correlate over a larger time span. Figure 10 proves the correctness of this assumption. The significance of the achieved relative control performance of up to $\approx 6 \mathrm{~dB}$ becomes clear when related to the maximum reduction in average acceleration power level of $\approx 12 \mathrm{~dB}$ in the $400 \mathrm{~Hz}$ third-octave band (not shown here). It can be stated that, in general, the largest improvements of the feedforward controller with long FIR filters occur for small numbers of reference sensors. The increase in relative control performance for seven and 10 reference sensors in the 400 and $500 \mathrm{~Hz}$ third-octave bands are presumably due to the higher number of dominant virtual noise sources and/or the suboptimal positioning of the smaller subsets of the reference sensors. The positive effect of seven and 10 reference sensors in the 100 and $125 \mathrm{~Hz}$ third-octave bands might be attributed to the longer FIR filters that achieve a better modeling of the lightly damped low-frequency modes.

If the reverberation room is excited by means of 10 uncorrelated sound sources, the dependency of the eigenvalue levels on sample length is largely reduced compared to the previous case with only one sound source. The results are shown in Fig. 11. However, the characteristics of an ideal diffuse sound field are still not reached, which again is in accordance with the spatial coherence shown in Fig. 3(b). The

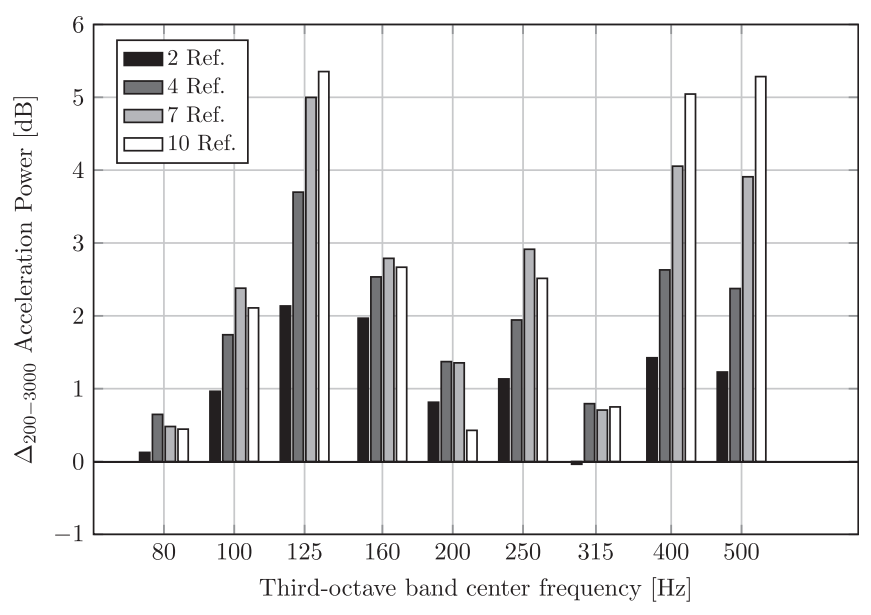

FIG. 12. Measured difference in control performance of a feedforward controller with 3000 and with 200 FIR filter taps dependent on the number of reference sensors with 10 uncorrelated sound sources in the reverberation room. impact on the relative feedforward control performance is shown in Fig. 12. As in the previous case, the achieved relative control performance of up to $\approx 5 \mathrm{~dB}$ is significant, since the maximum reduction in average acceleration power level amounts to $\approx 10 \mathrm{~dB}$ in the $315 \mathrm{~Hz}$ third-octave band (not shown here). In contrast to the previous case, the largest improvements in relative control performance occur for higher numbers of reference sensors. This is due to the fact that, compared to the previous case, the number of dominant virtual noise sources has increased but still lies below the number of dominant virtual noise sources of an ideal diffuse field. It can be assumed that, if the diffuse sound field was excited by a greater number of independent noise sources, the relative control performance would decrease for seven and 10 reference sensors as well.

\section{CONCLUDING REMARKS}

A theoretical discussion of the statistical properties of ideal diffuse sound fields reveals a close relationship between the spatial correlation and the spatial coherence. This relationship is verified for an ideal pure-tone diffuse sound field simulation model. Yet, the relationship is violated in the case of real, non-ideal diffuse sound fields generated in reverberation rooms. It is emphasized that the quality of a real diffuse sound field, which is defined according to its closeness of agreement to the ideal case, not only depends on the reverberation room's Schröder frequency but also on the number of statistically independent sound sources exciting the reverberation room. Whereas the spatial correlation approaches the theoretical curve for higher frequencies, the spatial coherence is strongly influenced by the number of independent sound sources and the chosen sample length or the spectral resolution, respectively. The dependency on sample length is explained by the reverberation time. If the sample length is small compared to the reverberation time, the spatial coherence of a non-ideal diffuse sound field converges to the ideal case. This has implications on the performance of active feedforward control, since the number of filter weights, and hence the sample length the filter uses for correlation, is a free design parameter. So, in the case of a non-ideal diffuse sound field excitation, the mutual coherence between reference sensors and the multiple coherence between reference and error sensors will depend on the chosen filter length. Therefore, the optimal number and position of reference sensors cannot 
be chosen independently from the control filter length. Furthermore, the control performance might be overestimated compared to the ideal case. This leads to the conclusion that the suitability of reverberation rooms used as disturbance excitation for the evaluation of active structures with feedforward controllers is limited. Depending on the application scenario of the test specimen, the diffuse sound field needs to be excited by a sufficient number of independent noise sources. Alternatively, a more realistic acoustic excitation might be achieved by means of a loudspeaker array placed in front of the structure. Future work will be concerned with the realization of acoustic excitations better suited for the evaluation of active systems. Also, the application of the presented methods and results regarding potential implications of non-ideal diffuse sound fields on active systems with feedback control laws or even on the transmission loss of passive structures might be of value.

${ }^{1}$ N. H. Schiller, "Decentralized control of sound radiation from periodically stiffened panels," Ph.D. thesis, Virginia Polytechnic Institute and State University, Blacksburg, Virginia, 2007.

${ }^{2}$ J. F. Wilby and F. L. Gloyna, "Vibration measurements of an airplane fuselage structure I. Turbulent boundary layer excitation," J. Sound Vib. 23(4), 443-466 (1972).

${ }^{3}$ J. F. Wilby and F. L. Gloyna, "Vibration measurements of an airplane fuselage structure II. Jet noise excitation," J. Sound Vib. 23(4), 467-486 (1972).

${ }^{4}$ M. Misol, T. Haase, H. P. Monner, and M. Sinapius, "Active trim panel with improved sound transmission loss under stochastic acoustic excitation," Proc. of the 20th International Congress on Sound and Vibration (ICSV20), CD-ROM (2013).

${ }^{5} \mathrm{M}$. Misol and C. Hesse, Numerische und experimentelle Untersuchungen zur Optimierung der Sensorik von adaptiven Steuerungen an doppelwandigen Leichtbaustrukturen (Numerical and Experimental Investigation of Double-Walled Lightweight Structures for the Optimization of Sensors of Adaptive Feedforward Controllers (DLR, Braunschweig, 2012), pp. $1-115$.

${ }^{6} \mathrm{~F}$. Jacobsen and T. Roisin, "The coherence of reverberant sound fields," J. Acoust. Soc. Am. 108, 204-210 (2000).
${ }^{7}$ S. J. Elliott, C. Maury, and P. Gardonio, "The synthesis of spatially correlated random pressure fields," J. Acoust. Soc. Am. 117, 1186-1201 (2005).

${ }^{8}$ M. Schröder, "Die statistischen Parameter der Frequenzkurven von grossen Raeumen" ("The Statistical Parameters of the Frequency Curves of Large Rooms"), Acustica 4, 594-600 (1954).

${ }^{9} \mathrm{P}$. Gardonio and S. J. Elliott, "Smart panels for active structural acoustic control," Smart Mater. Struct. 13, 1314-1336 (2004).

${ }^{10}$ W. P. Engels, O. N. Baumann, S. J. Elliott, and R. Fraanje, "Centralized and decentralized control of structural vibration and sound radiation," J. Acoust. Soc. Am. 119, 1487-1495 (2006).

${ }^{11} \mathrm{P}$. Gardonio and N. Alujevic, "Double panel with skyhook active damping control units for control of sound radiation," J. Acoust. Soc. Am. 128, 1108-1117 (2010).

${ }^{12} \mathrm{C}$. Bao and J. Pan, "Experimental study of different approaches for active control of sound transmission through double walls," J. Acoust. Soc. Am. 102, 1664-1670 (1997).

${ }^{13}$ J. Pan and C. Bao, "Analytical study of different approaches for active control of sound transmission through double walls," J. Acoust. Soc. Am. 103, 1916-1922 (1998).

${ }^{14} \mathrm{P}$. Gardonio and S. J. Elliott, "Active control of structure-borne and airborne sound transmission through double panel," J. Aircr. 36, 1023-1032 (1999).

${ }^{15}$ R. K. Cook, R. V. Waterhouse, R. D. Berendt, S. Edelman, and M. C. Thompson, Jr., "Measurement of correlation coefficients in reverberant sound fields," J. Acoust. Soc. Am. 27, 1072-1077 (1955).

${ }^{16} \mathrm{P}$. Welch, "The use of fast Fourier transform for the estimation of power spectra: A method based on time averaging over short, modified periodograms," IEEE Trans. Audio Electroacoust. 15, 70-73 (1967).

${ }^{17}$ F. Fahy and P. Gardonio, Sound and Structural Vibration: Radiation, Transmission and Response (Academic, London, 2007), Chap. 3.2, p. 139.

${ }^{18}$ M. Möser, Technische Akustik (Technical Acoustics), 7th ed. (Springer Verlag, Berlin, 2007), p. 223.

${ }^{19}$ S. J. Elliott, Signal Processing for Active Control (Academic, London, 2001), Chap.5, pp. 237-244.

${ }^{20}$ J. Minkoff, "The operation of multichannel feedforward adaptive systems," IEEE Trans. Signal Process. 45, 2993-3005 (1997).

${ }^{21}$ J. S. Bendat, "Solutions for the multiple input/output problem," J. Sound Vib. 44, 311-325 (1976).

${ }^{22}$ M. Akiho, M. Haseyama, and H. Kitajima, "Virtual reference signals for active noise cancellation system," J. Acoust. Soc. Jpn. Sec. E 19(2), 95-104 (1998).

${ }^{23}$ G. K. F. Rabl, "Recursive solution to wiener's multi-channel time filtering," Astron. Astrophys. 270, 552-556 (1993). 